case. Therefore, the present high-speed response is a direct outcome of the reduced capacitance of the MSM photodiode. Measurement of the noise characteristics of the present circuit showed an equivalent input noise current of 10 pA $\mathrm{Hz}^{-1 / 2}$ over the frequency range from 10 to $500 \mathrm{MHz}$. This value obtained is comparable to the combination of $p-i-n / a m p .{ }^{5}$ Therefore, the limiting factor of the noise level in the present transimpedance preamplifier would be FET noise of the front end rather than the dark current of the MSM diode.

In conclusion, a planar, monolithic integration of an MSM photodiode and a transimpedance preamplifier has been achieved by a process which is simple and compatible for use between optical and electric devices. The small capacitance characteristics of the MSM photodiode suitable for high-speed operations were pointed out. A 300-ps rise time response of the photoreceiver was demonstrated.
The authors are grateful to $T$. Touge and $K$. Dazai for encouragement. The present research is part of the largescale project "Optical measurement and control system" conducted under a program set up by the Agency of Industrial Science and Technology, Ministry of International Trade and Industry.

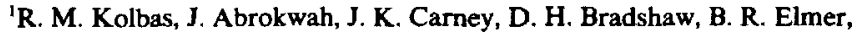
and J. R. Biard, Appl. Phys. Lett. 43, 821 (1983).

${ }^{2}$ S. Miura, O. Wada, H. Hamaguchi, M. Ito, M. Makiuchi, K. Nakai, and T. Sakurai, IEEE Electron Device Lett. EDL-4, 375 (1983).

${ }^{3}$ S. Miura, H. Machida, O. Wada, K. Nakai, and T. Sakurai, Appl. Phys. Lett. 46, 389 (1985).

${ }^{4}$ M. Ito, O. Wada, K. Nakai, and T. Sakurai, IEEE Electron Device Lett. EDL-5, 531 (1984).

${ }^{5}$ O. Wada, H. Hamaguchi, S. Miura, M. Makiuchi, K. Nakai, H. Horimatsu, and T. Sakurai, Appl. Phys. Lett. 46, 981 (1985).

${ }^{6} \mathrm{M}$. Ito and $\mathrm{O}$. Wada (unpublished).

\title{
Vibration resistance, short coherence length operation, and mode-locked pumping in passive phase conjugate mirrors
}

\author{
Mark Cronin-Golomb \\ Ortel Corporation, 2015 West Chestnut Street, Alhambra, California 91803 \\ Joel Paslaski and Amnon Yariv \\ California Institute of Technology, Pasadena, California 91125
}

(Received 22 July 1985; accepted for publication 23 September 1985)

\begin{abstract}
Because the ring and semilinear passive phase conjugate mirrors use dynamic transmission holograms, they are insensitive to vibration and may be pumped with light of short coherence length or with picosecond mode-locked laser light. Experimental demonstrations of these modes of operation are described.
\end{abstract}

In this letter we point out certain useful features which are associated with the structure of some self-pumped photorefractive passive phase conjugate mirrors ${ }^{1-5}$ (PPCM's). These devices are vibration resistant and even tend to work better in the presence of vibration. In spite of the length of the writing times for holographic gratings in photorefractive crystals (often in the range of seconds), they are free in these circumstances of that chief drawback of holography, the requirement that submicron stability be maintained during writing. And while with the exception of the two-interaction (2IR) mirror ${ }^{3}$ they rely on self-induced oscillation in external cavities, the coherence length may be much shorter than the external round trip length. They may be pumped by modelocked laser light, in spite of the fact that the four beams which induce the oscillation are not simultaneously present in the interaction region. Potential applications are in vibration resistant holography, mode locking of lasers including semiconductor lasers, ${ }^{6}$ and in the construction of ring laser gyroscopes. ${ }^{7}$ Each of these effects is a result of the reliance on a transmission, not a reflection grating in the four-wave mixing process.
Consider first the ring ${ }^{4,5}$ PPCM [Fig. 1(a)]. In this device, the beam whose phase conjugate is to be produced (beam 2) passes through a photorefractive crystal such as $\mathrm{BaTiO}_{3}$ and is fed back to it via optical path length $L$ as beam 4 around an optical ring of transmittance $M$. This ring can be formed either with a pair of mirrors with reflectivity product $M$ or by a length of optical fiber. ${ }^{7}$ An oscillation beam builds up, starting at the crystal as beam 3 which then travels the same path length $L$ around the ring to the crystal and passes through it as the phase conjugate beam 1 . Beam 3 is formed by diffraction of beam 2 off a self-induced transmission grating. Hence, beams 2 and 3 are mutually coherent. Beam 4 is the same as beam 2 after traveling distance $L$ around the ring, and beam 1 becomes beam 3 after traveling distance $L$. Beams 4 and 1 are thus also mutually coherent. The formation of the four-wave mixing transmission grating is thus influenced neither by variation (vibration) in the ring length nor by the possibility that the coherence length of the pumping light may be shorter than the ring length. A similar argument shows that picosecond mode-locked light can also generate the transmission grating for the ring PPCM: the pulse 


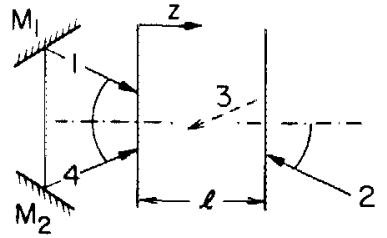

(a)

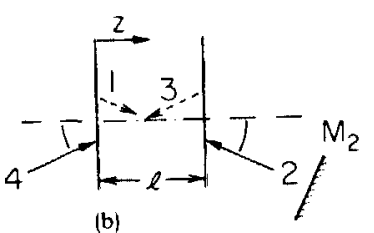

(b)

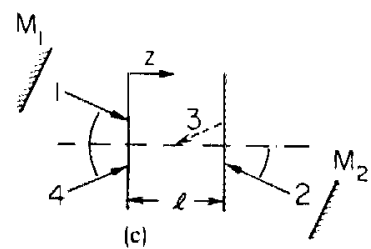

(c)
FIG. 1. Photorefractive passive phase conjugate mirrors. Both the ring mirror (a) and the semilinear mirror (b) are vibration insensitive and will work even if the coherence length of the incident radiation is less than the external cavity length. The linear mirror (c) is susceptible to vibration in the cavity length (but not of the crystal alone) and will not operate if the distance between the cavity mirrors is greater than the coherence length of the incident radiation. in beams 2 and 3 overlap since beam 3 is formed by diffraction from beam 2 , and the pulses in these beams travel identical distances around the ring to overlap again in the crystal as beams 4 and 1 . This flexibility has an upper limit discussed below that is set by the frequency selectivity of volume holograms.

In mathematical terms, the transmission grating is described by ${ }^{8}$

$$
g(z)=\gamma\left[A_{1}(z) A_{4}^{*}(z)+A_{2}^{*}(z) A_{3}(z)\right] / I_{0},
$$

where $A_{j}$ is the amplitude of beam $j, I_{j}=\left|A_{j}\right|^{2}$, $I_{0}=I_{1}+I_{2}+I_{3}+I_{4}$, and $\gamma$ is the photorefractive coupling constant. The differential equation governing the development of this grating in the $z$ direction derived from the coupled wave equations ${ }^{5,8,9}$ is

$$
\frac{d g}{d z}=\gamma g \frac{I_{1}+I_{2}-I_{3}-I_{4}}{I_{0}} .
$$

Neglecting diffractive effects, we have

$$
\begin{aligned}
& A_{1}(0)=A_{3}(0) e^{i k L}, \\
& A_{4}(0)=A_{2}(0) e^{i k L},
\end{aligned}
$$

which, when substituted into Eq. (1), yields

$$
g(0)=2 \gamma A_{2}(0)^{*} A_{3}(0) / I_{0}
$$

so that both the differential equation Eq. (2) and its boundary condition Eq. (4) are independent of cavity length $L$. This grating is therefore not influenced by vibration or shortness of coherence length. The absolute phase $\varphi$ of the reflection will, however, depend on the path length $L: \varphi \rightarrow \varphi+k L$. This is important in certain interferometric applications. ${ }^{10}$

The reflection grating, though not required for the PPCM, will build if the coherence length is greater than $L$ and if the system is not subject to vibration. It is described by $^{5}$

$$
g(z)=\gamma\left[A_{1}(z) A_{3}(z)^{*}+A_{2}(z)^{*} A_{4}(z)\right] / I_{0} .
$$

When Eq. (3) is substituted into Eq. (5) we find that

$$
g(0)=\gamma\left[\left|A_{3}(0)\right|^{2}+\left|A_{2}(0)\right|^{2}\right] e^{i k L} / I_{0},
$$

indicating regular holographic vibration sensitivity at the plane $z=0$. In the first experiment described below, we observed that the presence of the reflection grating was damaging to the overall phase conjugate reflectivity, and by washing this grating out by vibration, or by using short coherence length light, we could realize significant improvements. Analysis of the gratings formed between counterpropagating pairs of beams (1-2) and (3-4) yields path length dependences similar to those of the reflection grating.

Similar calculations for the semilinear ${ }^{2,5}$ PPCM [Fig. 1(b)] show that its transmission grating is also vibration insensitive, while its refiection grating washes out with vibra- tion. The PPCM with the lowest coupling constant threshold $^{2,5}$ is the linear PPCM [Fig. 1(c)]. It pays for this distinction in that all of its gratings are sensitive to cavity length vibration. The transmission grating is nevertheless still unaffected by vibration of the crystal alone. Such vibration, since it destroys the reflection and counterpropagation gratings, tends to improve the phase conjugate reflectivity as in the case of the ring PPCM. The linear PPCM depends on coherent feedback between its cavity mirrors, and is thus susceptible to short coherence and small changes in cavity length.

An upper limit to the spectral width $\Delta \lambda$ of the sources in the PPCM's described above is imposed by the spatial bandwidth of a volume hologram given by ${ }^{11}$

$$
\Delta \lambda / \lambda=\Lambda_{g} / l,
$$

where $\Lambda_{g}$ is the grating wavelength and $l$ is the interaction length. In our case, $\Lambda_{g} \approx 1 \mu \mathrm{m}$ and $l \approx l \mathrm{~mm}$ so that the bandwidth is about $10 \AA$. This is consistent with previous observations $^{12}$ that $44 \AA$ bandwidth rhodamine $6 \mathrm{G}$ dye laser light was too broad to even induce the gratings responsible for the fanning effect ${ }^{13}$ in $\mathrm{BaTiO}_{3}$ (two-beam coupling amplification of light scattering from crystal defects). In spite of this limitation, we have observed simultaneous self-pumped. phase conjugation of six lines of all lines output of an argon ion laser. ${ }^{14}$ This is possible because this output consists of several lines, each with a narrow bandwidth producing a superposition of several distinct gratings. This combination is less prone to cause grating washout than a single line with excessive bandwidth, which would produce a superposition of closely similar gratings tending to average to zero.

An experimental test of vibration insensitivity in ring PPCM was performed using a poled crystal of $\mathrm{BaTiO}_{3}$ as the photorefractive medium. The position of one of the ring cavity mirrors was dithered sinusoidally by a piezoelectric mount so that the amplitude of vibration in $L$ was one wave-

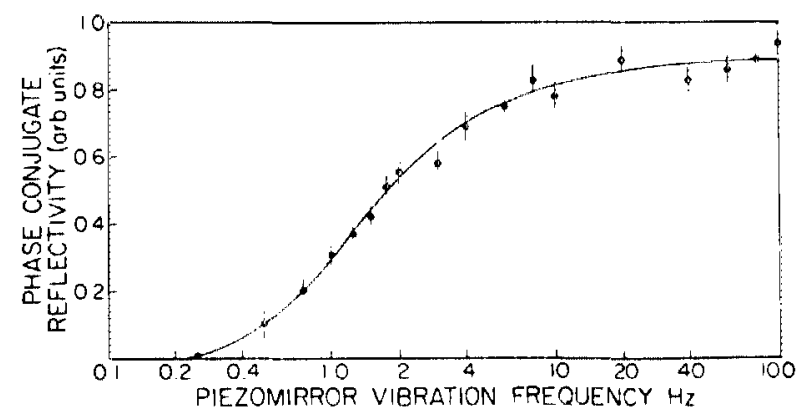

FIG. 2. Phase conjugate reflectivity of ring PPCM vs vibration frequency of piezomirror in feedback loop. Incident laser beam is single longitudinal mode at $514.5 \mathrm{~nm}$. Its intensity is $2 \mathrm{~W} / \mathrm{cm}^{2}$. The photorefractive four-wave mixing medium is $\mathrm{BaTiO}_{3}$. 
length of the laser light. Figure 2 shows the phase conjugate reflectivity as a function of vibration frequency of the piezomirror when single longitudinal mode 514.5-nm argon-ion laser light incident on the crystal with intensity $2 \mathrm{~W} / \mathrm{cm}^{2}$ was used. The phase conjugate reflectivity increases with increasing vibration frequency and stays at its maximum at frequencies greater than about $50 \mathrm{~Hz}$. The cut-on is centered at about $2 \mathrm{~Hz}$. Below $2 \mathrm{~Hz}$ the reflection and counterpropagation gratings could keep up with the changing path length since the grating response time at the intensity used was about $0.5 \mathrm{~s}$. Above $2 \mathrm{~Hz}$ these gratings washed out, leaving only the transmission grating, which was the only one required in the ring PPCM. With the laser running multilongitudinal mode, the loop length $(20 \mathrm{~cm})$ was longer than the coherence length, and the reflection and counterpropagating gratings again did not form. This time the reflectivity was independent of the vibration frequency of the piezomirror and equalled the reflectivity obtained in the single mode case at vibration frequencies greater than $50 \mathrm{~Hz}$.

The ring PPCM was also operated with a mode-locked dye laser using rhodamine $6 \mathrm{G}$ pumped by a mode-locked frequency-doubled $\mathrm{Nd}$ : YAG laser. This dye laser produced 5-ps pulses, occupying a spectral width of about $12 \AA$ FWHM centered around $591 \mathrm{~nm}$. The ring cavity length was $20 \mathrm{~cm}$ so that while the forward going pulses (in beams 2 and 3) overlapped in the crystal, as did the backward going pulses (in beams 1 and 4), these pairs did not appear in the crystal simultaneously. Nevertheless, oscillation built up almost as in the case of a cw input. The successive reinforcement of the grating by the forward and backward going pairs, together with the interpulse memory inherent in photorefractive holograms, was sufficient to generate the required grating. We note that the interpulse coherence of a true mode-locked laser is not necessary for this effect. The phase conjugate reflectivity was measured as a function of the feedback parameter $M$ controlled with a vibration attenuator inserted in the feedback loop. Data were taken for $M$ and 0 and 0.76 . Higher values of $M$ could not be reached because of feedback mirror losses and Fresnel losses at the loop side of crystal $(z=0)$. In this range, the reflectivity $R$ corrected for Fresnel reflections at the PPCM entrance face of the crystal $(z=l)$ and could be described by a linear relationship

$$
R=\alpha(M-\beta)
$$

where $\alpha=0.38$ and $\beta=0.15$. The maximum reflectivity, $23 \%$, was typical of reflectivities that can be obtained with cw lasers. Detailed studies of the operation of these PPCM's may be used to examine theories of short pulse grating formation in photorefractive crystals. ${ }^{15}$ Also, an advantage of the use of the ring PPCM as the end mirror for mode-locked lasers is that the colliding pulse geometry may be easily obtained by inserting a saturable absorber halfway around the ring where the pulses meet. The beams in the cavity are phase conjugates of each other, so that a focal point at the absorber, if required, may be easily accommodated. In a previous experiment, a two interaction region mirror ${ }^{3}$ was used as the end mirror of a synchronously pumped dye laser. ${ }^{16}$ There, the pulses were $15 \mathrm{ps}(5 \mathrm{~mm})$ long and so had approximately the same dimensions as the feedback path inside the crystal. Both pairs of pulses were therefore simultaneously present in the interaction region.

In summary, we have found that certain PPCM's including the ring mirror and the semilinear mirror are tolerant of vibration and short coherence length. They may be operated with mode-locked laser light, even though their external cavities are much longer than the width of the pulses. These effects hold promise for applications in robust holography, ring laser gyroscopes, and mode locking of lasers including dye lasers and semiconductor lasers.

This research was supported by the U. S. Army Research Office, Durham, NC.

'J. O. White, M. Cronin-Golomb, B. Fischer, and A. Yariv, Appi. Phys. Lett. 40, 450 (1982).

${ }^{2}$ M. Cronin-Golomb, B. Fischer, J. O. White, and A. Yariv, Appl. Phys. Lett. 41, 689 (1982).

${ }^{3}$ J. Feinberg, Opt. Lett. 7, 486 (1982).

${ }^{4}$ M. Cronin-Golomb, B. Fischer, J. O. White, and A. Yariv, Appl. Phys. Lett. 42, 919 (1983).

${ }^{5} M$. Cronin-Golomb, B. Fischer, J. O. White, and A. Yariv, IEEE J. Quantum Electron. QE-20, 12 (1984).

${ }^{6}$ M. Cronin-Golomb, K. Y. Lau, and A. Yariv, Appl. Phys. Lett. 47, 567 (1985).

${ }^{7}$ B. Fischer and S. Sternklar, Appl. Phys. Lett. 47, 1 (1985).

${ }^{8}$ B. Fischer, M. Cronin-Golomb, J. O. White, and A. Yariv, Opt. Lett. 6, $519(1981)$.

'M. Cronin-Golomb, B. Fischer, J. O. White, and A. Yariv, Opt. Lett. 7. 313 (1982).

${ }^{10} \mathrm{~J}$. Feinberg, Opt. Lett. 8, $569(1983)$.

"H. Kogelnik, Bell Syst. Tech. J. 48, 2909 (1969).

${ }^{12}$ R. McFarlane and D. G. Steel, Opt. Lett. 8, 208 (1983).

13. Feinberg, J. Opt. Soc. Am. 72, 46 (1982).

${ }^{14}$ M. Cronin-Golomb, S. K. Kwong, and A. Yariv, Appl. Phys. Lett. 44, 727 (1984).

${ }^{15} \mathrm{G}$. Valley, IEEE J. Quantum Electron. QE-19, 1637 (1983).

${ }^{16}$ R. K. Jain and K. Stenersen, Opt. Lett. 9, $546(1984)$. 